\title{
Characteristics of the Adherence to the Treatment in Patients and Its Dependence on the Type of Their Temperament
}

\section{IR Mavlyanov ${ }^{1}$, SI Mavlyanov ${ }^{1}$ and AB Soliev ${ }^{1,2 *}$}

${ }^{1}$ The Republican scientific and practical center of sports medicine at the National Olympic Committee of Uzbekistan, Uzbekistan

${ }^{2}$ Turin Polytechnic University in Tashkent, Kichik Khalka yuli str., Tashkent, Uzbekistan

*Corresponding Author: AB Soliev, The Republican scientific and practical center of sports medicine at the National Olympic Committee of Uzbekistan, Uzbekistan.

Received: June 11, 2019; Published: July 02, 2019

DOI: 10.31080/ASMS.2019.03.0337

\begin{abstract}
The article shows an analysis of the degree of adherence to the treatment of patients depending on the age category of patients. The results of the analysis indicate that there is a certain dependence of the degree of adherence on the age of patients. The structure of the reasons for the irregular intake of a drug depending on the age categories is also shown. The survey was conducted at the first Clinics of the Tashkent Medical Academy in 2017-2018.
\end{abstract}

Keywords: Adherence to the Treatment; Age Category; Patients; Temperament

\section{Introduction}

Today, no doubt that insufficient adherence to the treatment of chronic diseases is the main reason for reducing the effectiveness of the treatment, developing complications of the underlying disease, reducing the quality of life of patients and, consequently, increasing the cost of treatment [6]. Research has shown that longterm adherence to any treatment of any disease does not exceed $50 \%$ [2]. In a series of studies conducted on adherence to treatment, it was shown that the degree of adherence ranged from 43$78 \%[3,5,10,11]$. It has been established that $1 / 3$ of the patients discontinue the recommended treatment within 12 months after a cardiovascular event. Wherein, low treatment adherence was associated with a significant increase in the risk of cardiovascular complications $[1,3,4,7,8]$. Adherence to pharmacotherapy depends on many factors. One of the factors that may play an important role in assessing adherence is the patient's behavioral characterization.

Taking into account the above-mentioned data, the purpose of this study was to investigate the degree of adherence to treatment in patients depending on their type of temperament.

\section{Materials and Methods}

In this work, the results of a survey of 195 patients with chronic diseases of different age groups and gender were subjected to analysis. A questionnaire was used to assess the adherence and factors affecting them, including the Morisky-Green test (a validated test consisting of four questions evaluating adherence to treatment) [9] and 10 questions to identify the cause of acceptance or nonacceptance of a therapy. According to the Morisky-Green test, patients who scored four points were considered as adherent, three points as insufficiently adherent, and two or less as not adherent to treatment. The gender survey were divided into groups of men and women, as well as by age into the following age categories: up to 20 years old, 21 - 30 years old, 31 - 40 years old, 41 - 50 years old, 51 60 years old, 61 - 70 years, 71 - 80 years and over 81 years. To study and evaluate the behavioral characteristics, in particular the type of patient's temperament, the Eysenck test questionnaire was used.

The obtained data were processed by the method of variation statistics. 


\section{Results and Discussions}

The distribution of patients who were subjected to questioning by age categories is presented in Figure 1. As can be seen from the presented data, among the studied patients who were up to 20 years old was only $1.03 \%$. The same picture was observed in patients older than 81 years old, whose proportion was only $2.5 \%$. The rest of the patients were distributed in age groups from 21 to 80 years old. At the same time, the highest proportion of patients were in the age groups from 31 to 60 years old.

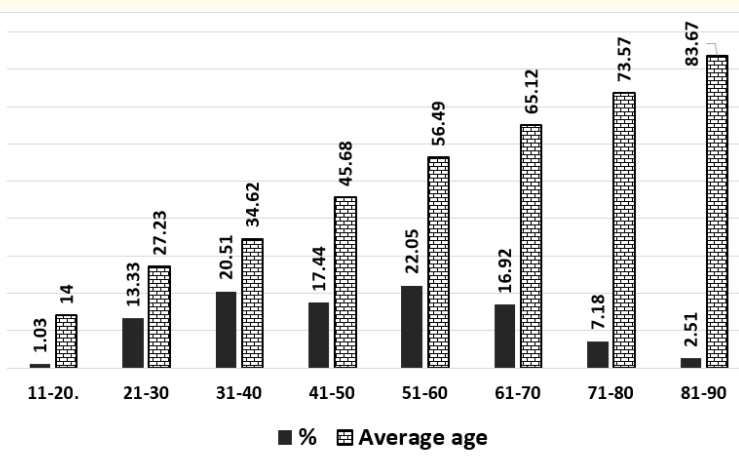

Figure 1: The distribution of patients by age categories.

It should be noted that in the age categories of 31-40 years and 51-60 years there was almost the same number of patients (21 and $22 \%$ of patients). A similar picture took place in the age categories of $41-50$ years and $61-70$ years ( $17.4 \%$ and $17 \%$, respectively).

The results of the analysis of adherence depending on the specified age category are presented in Figure 2.

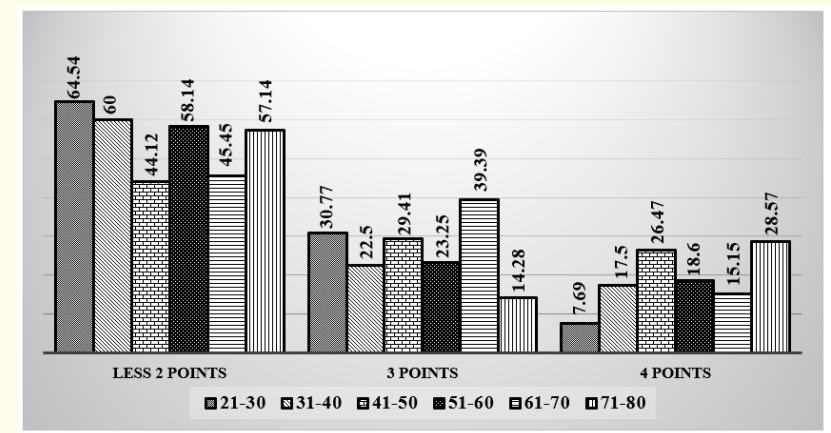

Figure 2: The degree of adherence in patients depending on the age categories (in \%).
As can be seen from Figure 2, the highest proportion of patients with non-adherence to treatment ( 2 or less points) is observed among patients of 21-30 years old. The proportion of such patients in this age group was about $65 \%$. A similar picture, but less pronounced, is noted among patients of 31-40 years, 51-60 years, and 71-80 years old. At the same time, the lowest proportion of patients with a lack of adherence to treatment ( 2 or less points) occurred among patients of $41-50$ years and $61-70$ years old ( $44 \%$ and $45 \%$, respectively).

The analysis of the frequency of occurrence of patients with unsatisfactory adherence to treatment ( 3 points) depending on the age category has shown that this proportion is high among patients aged 61-70 years old, reaching almost 2/5 of all surveyed patients, while in other age categories, this proportion ranged from $1 / 3$ to $1 / 7$ (Figure 2). At the same time, the lowest proportion of such patients was among patients of 71-80 years old.

As can be seen from the data presented in Fig. 2, the proportion of patients with satisfactory adherence to treatment (4 points) as a whole, regardless of the age group, is significantly low than the proportion of patients with unsatisfactory and lack of adherence to treatment and was ranged from $1 / 12$ up to $1 / 4$ parts of all respondents. Wherein, if among patients of 21 years to 50 years old this case gradually increases from $7.7 \%$ to $1 / 4$ parts of respondents, then from 51 to 70 years old again begins to decline to $1 / 7$ part of the studied patients. Consequently, the proportion of patients with adherence to treatment is high among the patients aged 41 - 50 years old (Figure 2).

In order to clarify the causes of non-compliance with medical prescriptions for treatment, we separately analyzed the results of a survey to identify the causes of irregular acceptance or nonacceptance of a therapy prescribed by a doctor. The results of this analysis are presented in (table 1).

As can be seen from the data presented in the table 1, the proportion of people whose reason for the irregular fulfillment of doctor's prescriptions was associated with the inability to buy drugs at a drugstore was the highest (60-61.5\%) among patients aged from 61 to 70 years old and from 71 to 80 years old, respectively. The lowest was among patients aged from 31 to 40 years old (46.8\%) and from 51 to 60 years old (52.8\%). In the remaining age categories, this reason was encountered quite often (Table 1). 
Characteristics of the Adherence to the Treatment in Patients and Its Dependence on the Type of Their Temperament

\begin{tabular}{|c|c|c|c|c|c|c|}
\hline \multirow{2}{*}{ Reasons } & \multicolumn{5}{|c|}{ Age categories (years old) } & 13 \\
\hline & 21-30 & $31-40$ & 41-50 & $51-60$ & 61-70 & 71-80 \\
\hline Cannot always buy the drug at the drug store & 58.33 & 46.90 & 58.10 & 52.80 & 60.00 & 61.50 \\
\hline Thinking of unnecessity to be treated & 25.00 & 28.10 & 35.50 & 38.90 & 46.70 & 61.50 \\
\hline Forgetting to take the drug & 51.20 & 53.10 & 48.40 & 72.20 & 50.00 & 53.80 \\
\hline Unwillingness to take the drug constantly & 54.20 & 65.60 & 48.40 & 30.60 & 70.00 & 46.10 \\
\hline Stopped taking medications due to normalization of BP & 41.70 & 40.60 & 41.90 & 36.10 & 63.30 & 38.50 \\
\hline Sometimes missed reception time, and reception at another time is inconvenient & 66.70 & 43.70 & 38.70 & 50.00 & 60.00 & 92.30 \\
\hline Doctor at the place of residence changed the drug & 33.33 & 40.60 & 48.40 & 50.00 & 40.00 & 38.50 \\
\hline Relatives advised to reduce the number of pills taken & 25.00 & 40.60 & 35.50 & 30.60 & 40.00 & 30.80 \\
\hline An allergic reaction has occurred. & 29.20 & 25.00 & 22.60 & 16.70 & 20.00 & 30.80 \\
\hline Refusal of treatment without giving a reason & 33.33 & 15.60 & 12.90 & 22.20 & 26.70 & - \\
\hline
\end{tabular}

Table 1: Reasons for irregular admission/non-admission therapy depending on the age categories of patients (\%).

The proportion of people who consider no need to be treated had a tendency to increase as the age of patients increases. So, if this reason was found among patients aged from 21 to 30 years old in every fourth patient, among patients from 71 to 80 years old it reaches two out of three patients (Table 1 ).

Patients who forget taking the prescribed drug, among patients in almost all age categories, occur equally often, approximately in every second patient. The exception is patients aged from 51 to 60 years, where the frequency of occurrence of this cause reaches almost $3 / 4$ parts of the patients surveyed.

From $30.6 \%$ to $70 \%$ of patients compared by age do not always comply with the recommendations of the doctor because of the unwillingness to take medications (Table 1). The highest proportion of such patients are among the age group of 61 to 70 years old, and the lowest are among patients aged 51 to 60 years old. Patients who stop taking the drug due to the normalization of blood pressure levels are high, as in the previous case, among patients aged 61 to 70 years old.

As can be seen from table 1, the frequency of occurrence among the analyzed groups of patients with irregular implementation of the doctor's recommendation for taking medications associated with skipping the time of admission due to the inconvenience of their admission at another time is quite high. In the age groups of 21-30 years, 61-70 years and 71-80 years, the proportion of this reason is clearly high in half of the patients surveyed, and in the other age categories this cause is found in half or less of the respondents.
Such reasons as a doctor at the place of residence changed the drugs, relatives advised to reduce the number of pills taken, an allergic reaction appeared, the refusal of therapy without specifying the reason, in general, among the interviewed patients met with less frequency compared to the specified reasons (Table 1). Moreover, the difference between the studied age categories was also not so pronounced (Table 1). Consequently, in terms of the frequency of occurrence of individual causes of irregular drug intake, there are certain differences depending on the age categories, which is associated with the nature of the diseases often encountered in these age groups, and the role of gender differences in the structure of the diseases studied is not excluded.

For us, it was also of some interest to analyze the degree of adherence depending on the type of patients' temperament.

The results of this analysis are presented in figure 3.

As can be seen from the presented data, the highest incidence of patients with a satisfactory adherence to the therapy was observed among sanguine persons, and the unsatisfactory adherence was among choleric persons (Figure 3). At the same time, the lack of adherence to the pharmacotherapy was the highest among patients with the phlegmatic type of temperament (Figure 3). Consequently, patients with sanguine and choleric types of temperaments are more committed to the therapy compared to patients with phlegmatic and melancholic types of temperaments. 


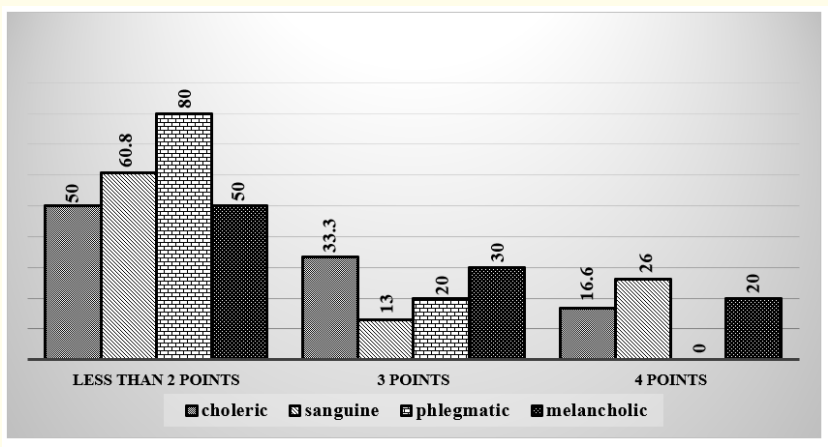

Figure 3: The degree of adherence in patients depending on their type of temperament (in \%).

We also analyzed the patient's adherence to therapy from the position of the type of the nervous system. The analysis showed that satisfactory adherence is almost often more frequent among extrovert patients, and a lack of adherence to therapy, on the contrary, is more common among introvert patients.

Thus, the results of the study indicate that regardless of the age of patients, in general, there is a fairly low level of adherence to treatment. In the frequency of occurrence of individual degrees of adherence to treatment, there is a certain dependence on the age category of patients. Among the reasons of irregular admission or non-admission of the drug and their frequency of occurrence, there is a certain dependence on the age of patients. There is also a certain dependence between adherence and the types of patients' temperament.

\section{Conclusions}

1. In patients with different age categories, persons who are adherent and not adherent to treatment are often found unequally.

2. The proportion of persons adherent to treatment among patients aged 41 to 50 years and 71 to 80 years is relatively high, while it is low among patients aged 21 to 30 years old.

3. Among the reasons of irregular intake of the drug most often occur, regardless of the age of patients, the reasons associated with the inability to buy the drug in the drug store, lack of desire to take the drug all the time, forgetfulness of taking the drug and periodic omissions of taking the drug.

4. Sanguine and extrovert patients are more adherent to the treatment.

\section{Conflict of Interest}

The authors declare that there is no conflict of interest.

\section{Bibliography}

1. Gusev EI., et al. "Adherence to primary therapy in patients with post-stroke depression". Journal of Neurology (in Russian) 4 (2011): 54-59.

2. Martsevich SY., et al. "Efficacy and safety of drug therapy in primary and secondary prevention of cardiovascular diseases". Journal of Rational Pharmacotherapy in Cardiology (in Russian) 5 (2011): 72.

3. Puchinyan NF., et al. "Adherence to the recommended treatment of patients undergoing acute coronary syndrome, and the risk of cardiovascular complications within a year after hospitalization". Journal of Rational Pharmacotherapy in Cardiology (in Russian) 5 (2011): 567-573.

4. Burt VL., et al. "Prevalence of hypertension in the US adult population. Results from the Third National Health and $\mathrm{Nu}-$ trition Examination Survey, 1988-1991". Hypertension 25.3 (1995): 305-313.

5. Claxton AJ., et al. "A systematic review of the associations between dose regimens and medication compliance". Clinical Therapeutics 23.8 (2001): 1296-1310.

6. Geest S.De and Sabaté E. "Adherence to long-term therapies: evidence for action". European Journal of Cardiovascular Nursing 2.4 (2003): 323.

7. Hershey JC., et al. "Patient compliance with antihypertensive medication". American Journal of Public Health 70.10 (1980): 1081-1089.

8. Lüscher TF., et al. "Compliance in hypertension: facts and concepts”. Journal of hypertension. Supplement 3.1 (1985): 3-9.

9. Morisky DE., et al. "Concurrent and predictive validity of a self-reported measure of medication adherence". Medical Care 24.1 (1986): 67-74.

10. Osterberg L and Blaschke T. "Adherence to medication". New England Journal of Medicine 353.5 (2005): 487-497.

11. Waeber B., et al. "Compliance with aspirin or placebo in the Hypertension Optimal Treatment (HOT) study". Journal of Hypertension 17.7 (1999): 1041-1045.

\section{Volume 3 Issue 8 August 2019}

(C) All rights are reserved by AB Soliev., et al. 